\title{
Técnica para elaborar férulas de mano en isopor y madera terciada
}

\author{
Woodruff Thomforde, David.
}

\section{- Resumen}

Se ha desarrollado en Paraguay una técnica para hacer férulas de mano en isopor (o "Plumavit"), madera terciada y papel. La plancha de isopor se corta para cubrir la superficie de la palma y el antebrazo y se pega a la madera terciada para proporcionar resistencia. Se encolan pedazos de papel encima del isopor y luego se pintan con pintura al aceite. Se utiliza velcro para elaborar las correas. Las férulas realizadas incluyen una férula de posicionamiento para una mano fláccida, una férula separadora de dedos para una mano espástica, una férula de posicionamiento para un paciente con acortamiento de los tendones flexores de la muñeca, un abductor del pulgar para un dedo pulgar débil pero funcional, un abductor del pulgar para un pulgar no funcional, una férula de posicionamiento de muñeca (cock-up) y una férula de posicionamiento para una persona con artritis reumática para prevenir la desviación cubital.

Se comparan las ventajas y desventajas de esta técnica con otras alternativas de materiales para férulas ( $P V C$, metal, venda enyesada, cuero, bambú y polipropileno). Las mayores ventajas de esta técnica son el bajo costo, la alta adaptabilidad a la mano, el uso de técnicas conocidas por los artesanos locales, herramientas económicas y facilidad de reparación o modificación. Las desventajas mayores son la no-disponibilidad de materiales en algunos países, la necesidad de desarrollar nuevas relaciones entre terapeutas y artesanos locales y la dificultad para una completa desinfección. Las futuras aplicaciones de esta técnica para elaborar férulas dinámicas de manos y pies se están discutiendo. Esta técnica promete expectativas en países de bajo ingresos como Paraguay.

\begin{abstract}
- Abstract
A technique has been developed in Paraguay for making hand splints from styrofoam, plywood and paper. Sheet styrofoam is cut to fit the palmar surface of the forearm and glued to plywood to provide strength. Pieces of paper are glued over the styrofoam and then painted with oil paint. Velcro is used to make straps. The splints made thus far include a resting hand splint for a flaccid hand, a finger-spreader splint for a spastic hand, a resting hand splint for a patient with shortening of the wrist flexors, a thumb support splint for a weak but functional thumb, a thumb positioning splint for a non-functional adducted thumb and a resting hand splint for a person with rheumatoid arthritis to prevent ulnar deviation

The advantages and disadvantages of this technique are compared with those of other alternative splinting materials (PVC, metal, plaster of Paris bandage, leather, bamboo and polypropylene). The major advantages of this technique are low cost, high adaptability to the individual hand, use of techniques known by local artisans, low-cost tools and ease of repair or modification. The major disadvantages are non-availability of materials in some places, need to develop new relationships between therapists and local artisans and difficulty with thorough disinfecting. Future applications of the techniques for making dynamic hand splints and foot splints are discussed. This technique appears to have promise in low to middle income countries such as Paraguay.
\end{abstract}

\footnotetext{
."Técnica para elaborar ferulas de mano en isopor y madera terciada".Contacto > > Aca Caraya 642 Asunción, Paraguay. Starbuck St 13556, Whittier, CA 90605 EE.UU e mail: dtandkh@hotmail.com
} 


\section{Introducción}

Uno de los mayores problemas que enfrentan los terapeutas ocupacionales en los países en vías de desarrollo como Paraguay son los materiales apropiados para hacer las férulas de la mano. Se usan los termoplásticos de baja temperatura en los países desarrollados para hacer las férulas de calidad superior en un tiempo corto pero son demasiado caras para la mayoría de los pacientes en los países menos adinerados. Los termoplásticos de baja temperatura requeridos para elaborar una férula de posicionamiento de mano costarían ligeramente menos al equivalente del sueldo de una semana de un obrero con salario mínimo en Paraguay, o alrededor de 40 dólares estadounidenses.

Los terapeutas ocupacionales y otros rehabilitadores alrededor del mundo están usando los materiales alternativos como la venda enyesada, cloruro polivinílico (PVC), metal, cuero, bambú y polipropileno dependiendo de lo que está localmente disponible. Algunas férulas excelentes son realizadas con estos materiales, pero ellas también tienen sus inconvenientes.

Las férulas en venda enyesada son baratas (en Paraguay, alrededor de 2 dólares estadounidenses) y pueden hacerse en 20 minutos por un Terapeuta Ocupacional con experiencia utilizando las herramientas extensamente disponibles. Estas férulas pueden estar hechas para calzar bien en la mano del paciente y pueden ser resistentes al momento de fabricación. Pero son pesadas, no son fáciles de limpiar y normalmente no duran por mucho tiempo. Es difícil modificarlas después de ser elaboradas.

Las férulas de PVC son hechas de 2-mm en hoja de PVC o de los tubos de PVC. Ellas son fáciles limpiar, son ligeras, resistentes y duran un largo tiempo. Los tubos de PVC son baratos y extensamente disponibles. Las hojas de PVC no están disponibles fácilmente y son más caras. El material de la hoja de PVC es más fácil de moldear que el material del tubo. Una vez dominado el proceso para trabajar con cualquier material, puede realizarse las férulas rápidamente. Las férulas pueden hacerse utilizando un horno de cocina y herramientas simples. El material debe calentarse para ser moldeado, para lo cual debe ponerse en un molde de yeso de la mano (esto complica el proceso de fabricación) o en la mano completamente envuelta del terapeuta o del paciente (lo cual limita el grado en el que la férula puede encajar en la mano del paciente). Además, las habilidades necesarias para trabajar con PVC son raras o están ausentes en la mayoría de las comunidades por lo tanto el Terapeuta Ocupacional tendrá que hacer las férulas él mismo o entrenar a otros en algunas tareas. Las férulas no pueden modificarse después de que son hechas. En la mayoría de los casos, son los técnicos quienes fabrican estas férulas con la guía de los terapeutas ocupacionales.

Las férulas hechas en planchas de metal reciclado o en pedazos de estaño pueden ser muy baratas y ligeras. Estos materiales se golpean para formar el molde de la mano del paciente. Algunas comunidades en diferentes culturas pueden tener artesanos con estas habilidades. Las herramientas necesarias se encuentran disponibles en la mayoría de las comunidades. A menos que las férulas estén muy bien hechas, no son lo suficientemente resistentes. Las mismas también no pueden formarse directamente en la mano del paciente, deben martillarse de una determinada forma y luego colocarse en la mano del paciente, por lo tanto no siempre encajan bien. Estas férulas son raras, y normalmente no son hechas por terapeutas ocupacionales.

Las férulas de bambú son ligeras, baratas y fáciles limpiar. En La mayoría de las comunidades donde crece el bambú se cuenta con artesanos locales con las habilidades y herramientas necesarias para elaborar las férulas quienes son entrenados por un Terapeuta Ocupacional. Pero la maleabilidad del bambú es limitada por lo tanto la férula no encajará bien en la mano. El bambú que puede doblarse muchas veces no será lo suficientemente resistente para una férula. 
Las férulas de cuero son ligeras, baratas y pueden durar un largo tiempo. La mayoría de las comunidades tienen artesanos que trabajan el cuero con las herramientas y habilidades necesarias. El cuero debe ponerse húmedo en la mano del paciente, debe ser atado herméticamente y dejarlo allí durante varias horas hasta que seque; entonces este método no es apropiado para las manos espásticas o dolorosas. Las férulas no son fáciles de limpiar. El cuero necesita un refuerzo de metal para ser resistente. Estas férulas están hechas normalmente por artesanos de la comunidad.

Las férulas de polipropileno son fáciles de limpiar, livianas, resistentes y duran un largo tiempo. Sin embargo son caras (una férula de mano puede costar más de 3 semanas de sueldo de un obrero de salario mínimo en Paraguay), requieren equipo de calentamiento sofisticado y las habilidades necesarias no están disponibles en muchas comunidades, y no pueden modificarse luego de ser realizadas. En la mayoría de los casos, los técnicos ortopédicos hacen estas férulas.

Además, en mi observación, las Férulas realizadas con PVC, venda enyesada y polipropileno ponen el dedo pulgar en extensión, éste queda en el mismo plano de la mano. Las características físicas de los materiales pueden promover esto.

Yo he estado experimentando con una nueva técnica que combina el isopor (para crear una forma que se amolde a la mano y antebrazo), madera terciada (para darle resistencia) y papel (para crear una superficie lisa). Se usan las correas de velcro para atar la férula a la mano del paciente. Durante los últimos 2 años, he elaborado más de 40 férulas, con un proceso gradualmente evolucionado.

He desarrollado siete modelos diferentes:

- una férula de posicionamiento para una mano fláccida,

- una férula separadora de dedos para una mano espástica,

- una férula de posicionamiento de mano para un paciente con acortamiento de los tendones

flexores de la muñeca,

- un abductor del dedo pulgar para un dedo pulgar débil pero funcional,

- un abductor del dedo pulgar para un dedo pulgar no funcional,

- una férula de posicionamiento de mano para una persona con artritis reumática para prevenir la desviación cubital,

- una férula de posicionamiento de muñeca (cock-up),

El costo de los materiales va de 50 centavos a 2.5 dólares estadounidenses en Paraguay. El tiempo necesario para realizar las férulas varía de 30 minutos a $21 / 2$ horas. Los pacientes han estado utilizando varias ediciones de estas férulas por más de 1 1/2 años con resultados satisfactorios. Durante los últimos tres meses, he entrenado a 24 profesionales y técnicos en este proceso. Actualmente uno de ellos ha comenzado la producción de las férulas independientemente.

\section{- Procedimiento}

Los siguientes son los pasos a seguir para realizar una férula de posicionamiento de mano para un adulto (los pasos para un niño son los mismos, pero se usa el isopor más delgado):

1) Ponga la mano del paciente en un pedazo de papel y haga un trazo alrededor de la mano que diste 2/3 del antebrazo; marque el arco de las articulaciones metacarpo-falangicas (MCF), el arco de las articulaciones interfalangicas proximales (PIF), el pliegue palmar y donde las eminencias 
tenares y hipotenares hypotenares juntan (una distancia muy corta, distal del pliegue de la muñeca); los dedos deben estar juntos y el dedo pulgar separado.

2) Corte la forma en el papel, doble el dedo pulgar debajo y marque la forma en un isopor de $21 / 2$ centímetros de espesor; corte la forma con un cuchillo "cutter" (o "cartonero") pequeño.

3) Corte la forma en papel donde las eminencias tenares y hipotenares se juntan; trace la porción de la mano en un isopor de 5 centímetro de espesor y recorte la forma.

4) Coloque la mano en una posición relajada con la punta del dedo pulgar y la del índice casi tocándose; para un paciente con una mano fláccida, ésta será la posición de descanso (8) con la muñeca a 15-20 grados de extensión, las articulaciones MCF, PIF y interfalangicas distales (DIF) a 10-20 grados de flexión, el dedo pulgar en abducción y flexionado aproximadamente 10 grados con respecto al antebrazo; medir el ángulo antebrazo-metacarpo en ambos el cubital y el lado radial de la mano (yo uso el papel plegado ya que los goniómetros son raros en Paraguay); en el lado radial de la mano, esta figura puede usarse también para los ángulos de las articulaciones MCF, PIF y DIF, ya que ellos son muy similares en la posición de descanso; en el lado cubital de la mano, deben medirse independientemente los ángulos de las articulaciones MCF, PIF y DIF.

5) Marque el contorno de la mano en ambos lados del isopor de 5 centímetros de espesor, utilizando cada los ángulos de las articulaciones y las distancias entre las articulaciones medios en la mano trazada en el papel; use el cutter chico para dar forma al isopor conforme a la forma de la mano, asegurándose de mantener los arcos metacarpianos y carpianos y el arco longitudinal.

6) Marque el pliegue tenar sobre el isopor y corte el resto de isopor que se encuentre próximo al pliegue.

7) En otro modelo de papel, mencionado en el punto tres, desdoblar el pulgar y cortar a lo largo de la línea extendida en el papel en una dirección próxima a lejana hasta que el dedo pulgar casi se desuna completamente; coloque el modelo de papel en el isopor; coloque el dedo pulgar para que se encorve ligeramente respecto al antebrazo; marque la posición del dedo pulgar del lado del bloque de isopor; recorte la forma del dedo pulgar.

8) Ponga el molde de papel sobre el modelo de isopor para asegurarse de que sea la forma correcta; si el paciente está disponible, ponga el isopor bajo su palma; haga las modificaciones necesarias para lograr la posición correcta.

9) Durante el proceso que se lleva a cabo para dar forma al isopor de 5 centímetros de espesor, el pedazo se acorta; por consiguiente, ponga el isopor de 5 centímetros de espesor sobre el isopor de $21 / 2$ centímetros de espesor de la mano y el antebrazo y corte el isopor de $2 \frac{1}{2}$ centímetros con la nueva forma.

10) Coloque el isopor modificado de $2 \frac{1}{2}$ centímetros de espesor sobre madera terciada de $9 \mathrm{~mm}$, dibuje y recorte la forma; lije los bordes; encole los dos pedazos de isopor juntos y la madera terciada debajo de ambos, usando cola especial para isopor.

11) Talle la porción del antebrazo de la férula hasta que quede ligeramente cóncava.

12) Cubra el isopor con una cola cocinada hecha de harina (mandioca, trigo o maíz) y agua; aplique pedazos pequeños de bolsas de papel mojado con la cola para cubrir la férula, aplanando cualquier mancha áspera. 
13) Después de que esto esté completamente seco, use la cola blanca para atar cualquier pedazo suelto de papel; mezcle partes iguales de cola blanca y agua y pinte el papel.

14) Después de que esto esté completamente seco, pinte la férula con por lo menos dos capas de pintura al aceite.

15) Encole el velcro al material que utilizará para atar para realizar tres correas; éstos se atan a la madera terciada con tornillos; las correas se atan utilizando anillos de metal.

Las otras férulas de la mano son variaciones de ésta. En la férula de separación de dedos el modelo de papel se traza con los dedos y el pulgar separados. Un bloque de madera se forma para encajar entre el dedo pulgar y el índice y se ata a la madera terciada. La muñeca se mantiene neutra. Se cortan pequeños pedazos de isopor para encajar entre los dedos.

En la férula de posicionamiento de la muñeca, la madera y la base de isopor están cortadas en el pliegue tenar y el pliegue distal de la palma, y se forma un bloque de madera para encajar bajo la palma y el relleno.

En la férula de posicionamiento de mano para un paciente con los tendones flexores de la muñeca acortados, la base de isopor y la madera terciada están cortadas en el pliegue de la muñeca, y se unen por un triángulo de madera al ángulo del dedo pulgar y del antebrazo. Además, debido a que la mano del paciente no puede apoyarse sobre un pedazo de papel para ser trazada, se realiza un molde de la mano del paciente en venda enyesada en la posición deseada y se toman todas las dimensiones y trazados del mismo.

En la férula de posicionamiento de mano para prevenir la desviación cubital se han acolchado los pedazos de madera terciada atados al lado cubital de los dedos y al lado radial de la muñeca para mantener la mano y el brazo en una línea recta.

El abductor del dedo pulgar para un pulgar funcional pulgar utiliza un proceso diferente. Se busca un objeto (o se realiza en arcilla), que se rellena el espacio entre el dedo pulgar y la palma permitiendo apretar. El objeto debe extenderse más allá de la articulación interfalángica del dedo pulgar, pero no más allá del pliegue de la articulación MCF del dedo índice. Esta forma se copia en el papel, y se extiende $1 / 2$ centímetro en todos sus lados, y luego un pedazo de isopor de 3 centímetros de espesor es cortado con esa forma. Los bordes que tocan el dedo pulgar y el espacio entre el pulgar y el dedo indicó se tallan para ser cóncavos con una profundidad de $1 / 2 \mathrm{~cm}$. Se encola el velcro macho en los lados y en el borde superior de la férula. Una tira de velcro hembra es cortada con el largo necesario para poder atar el dedo pulgar firmemente a la férula. Otro pedazo de velcro hembra se corta para poder atarse al lado radial de la férula, el mismo debe extenderse bajo el brazo y alrededor de la parte de atrás de la muñeca y se debe doblar alrededor de la muñeca y cruzar por la parte de atrás de la mano antes de atar al borde superior de la férula. La fricción del velcro sobre el brazo deja el dedo pulgar en abducción. Se aplican papel y pintura al isopor que quede a la vista.

La férula abductora de pulgar -para un pulgar no funcional- está hecha casi de la misma forma que la de arriba, pero el pedazo de isopor es más grande, ya que se extiende más allá de el pliegue de la articulación MCF del dedo índice y es 5 centímetros de gruesa, esto se realiza para proporcionar mayor estabilidad de la presión aductora. (Figuras 1-7) 
Fotografía 1 - Férula de posicionamiento para una mano fláccida

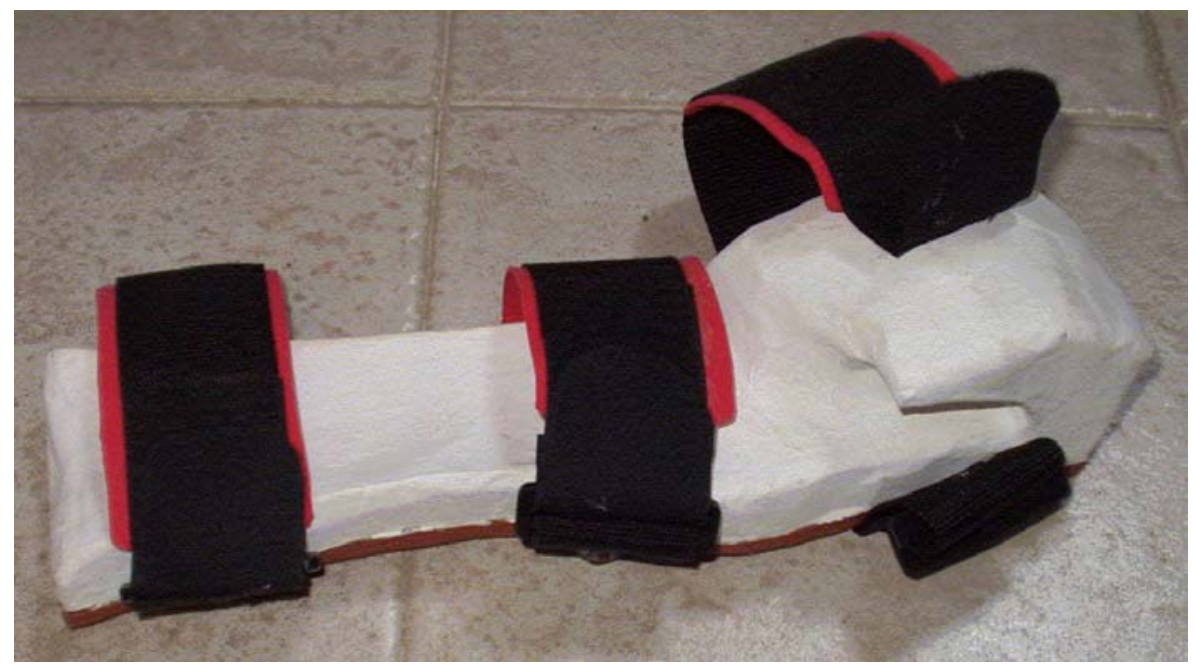

Fotografía 2 - Férula separadora de dedos para una mano espástica

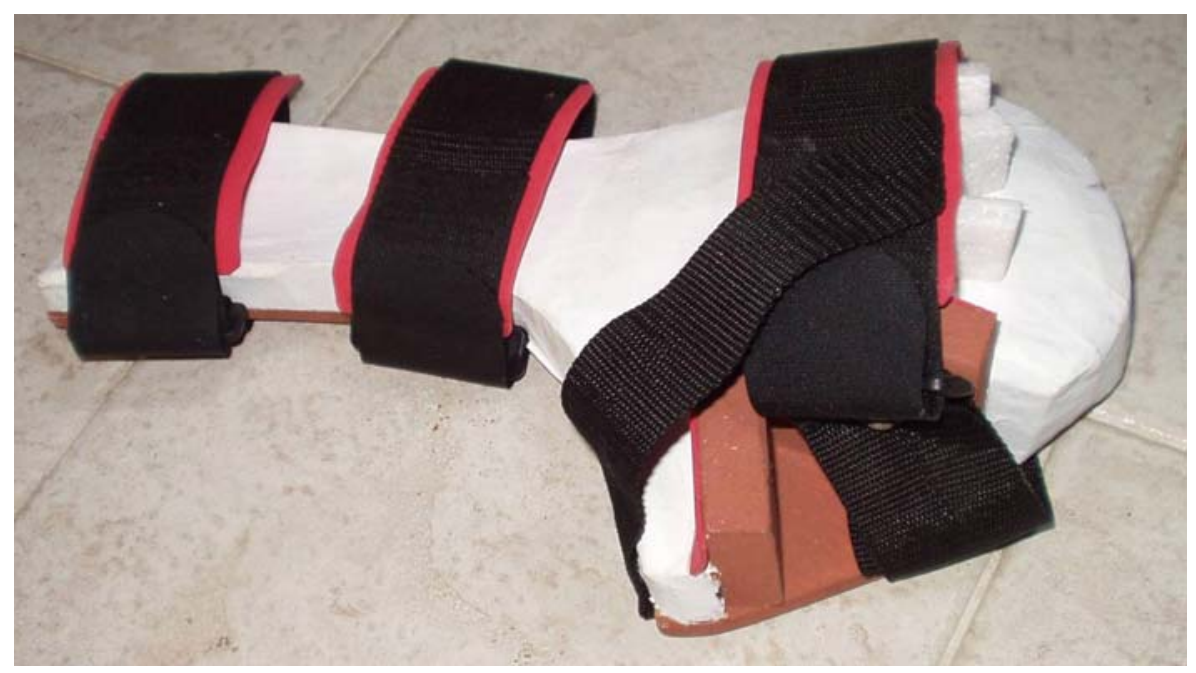


Fotografía 3 - Férula de posicionamiento de mano para un paciente con acortamiento de los tendones flexores de la muñeca

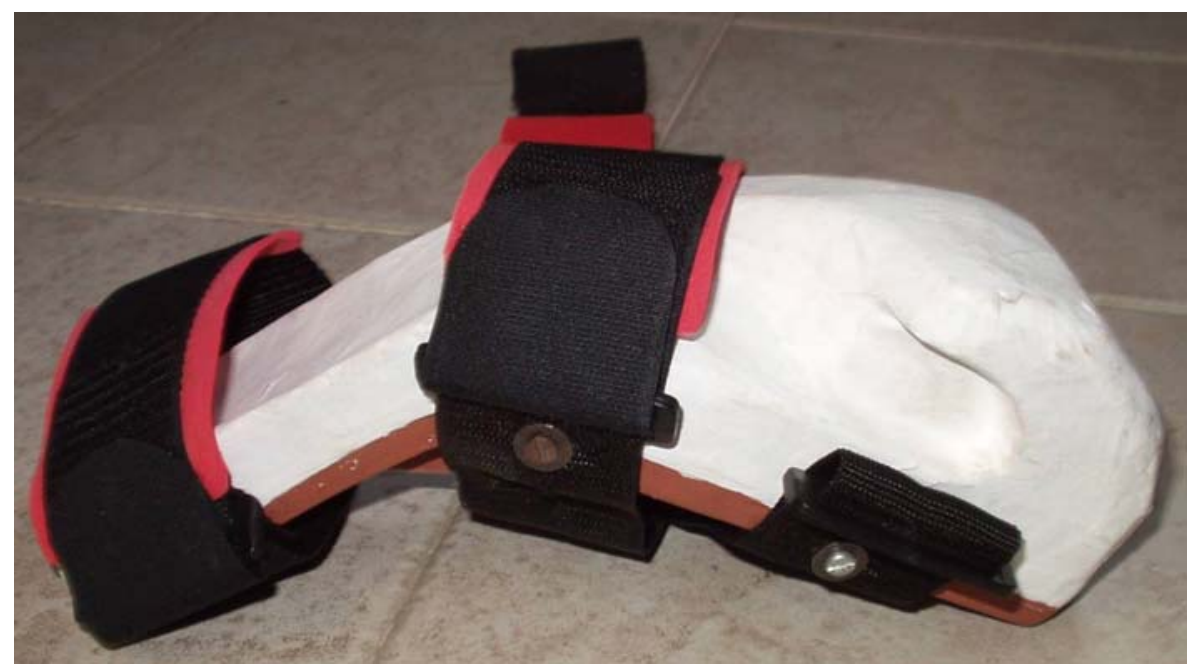

Fotografía 4 - Férula de posicionamiento de mano para una persona con artritis reumática para prevenir la desviación cubital

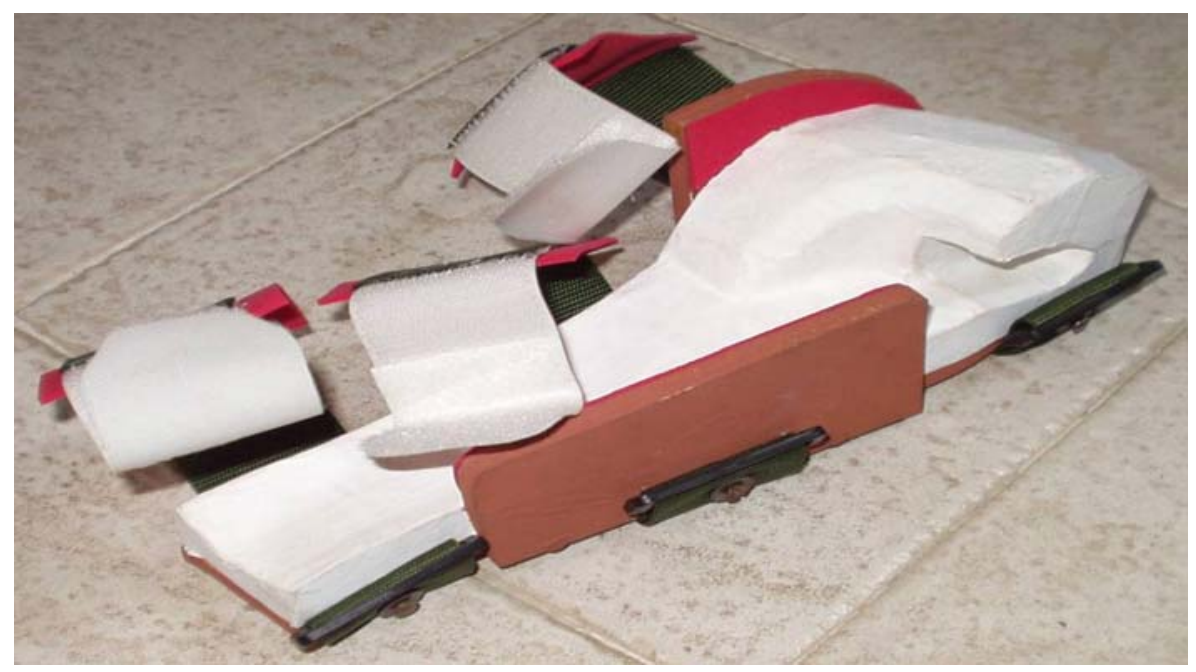


Fotografía 5 - Férula de posicionamiento de muñeca (cock-up)

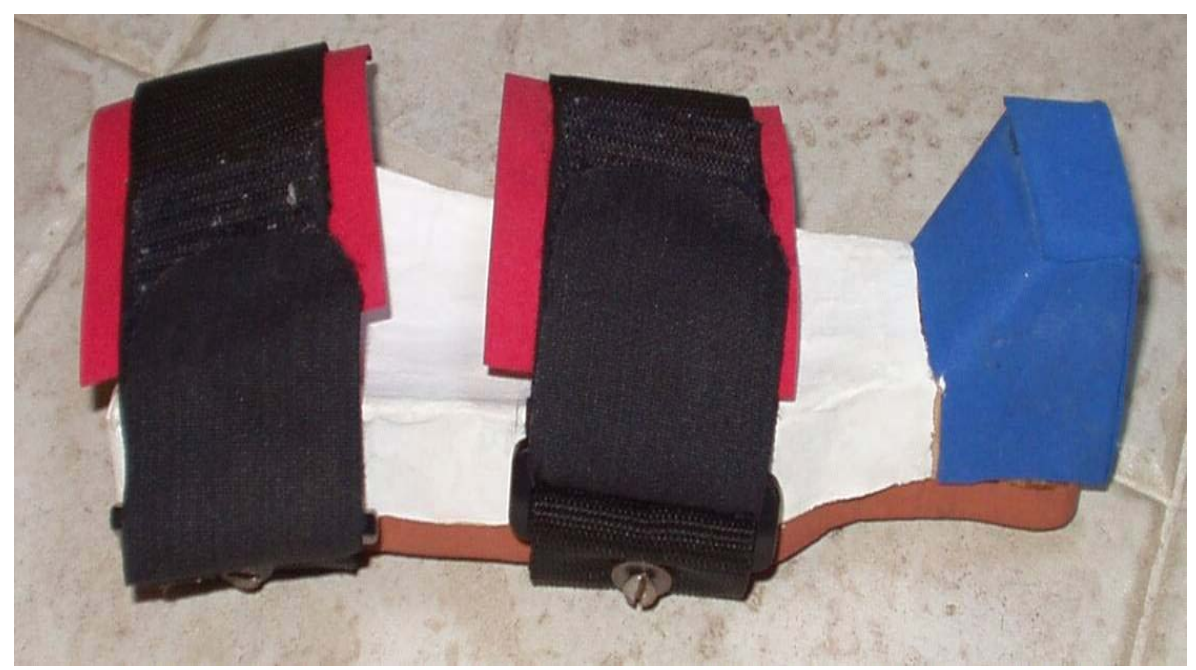

Fotografía 6 - Un abductor del dedo pulgar para un dedo pulgar débil pero funcional

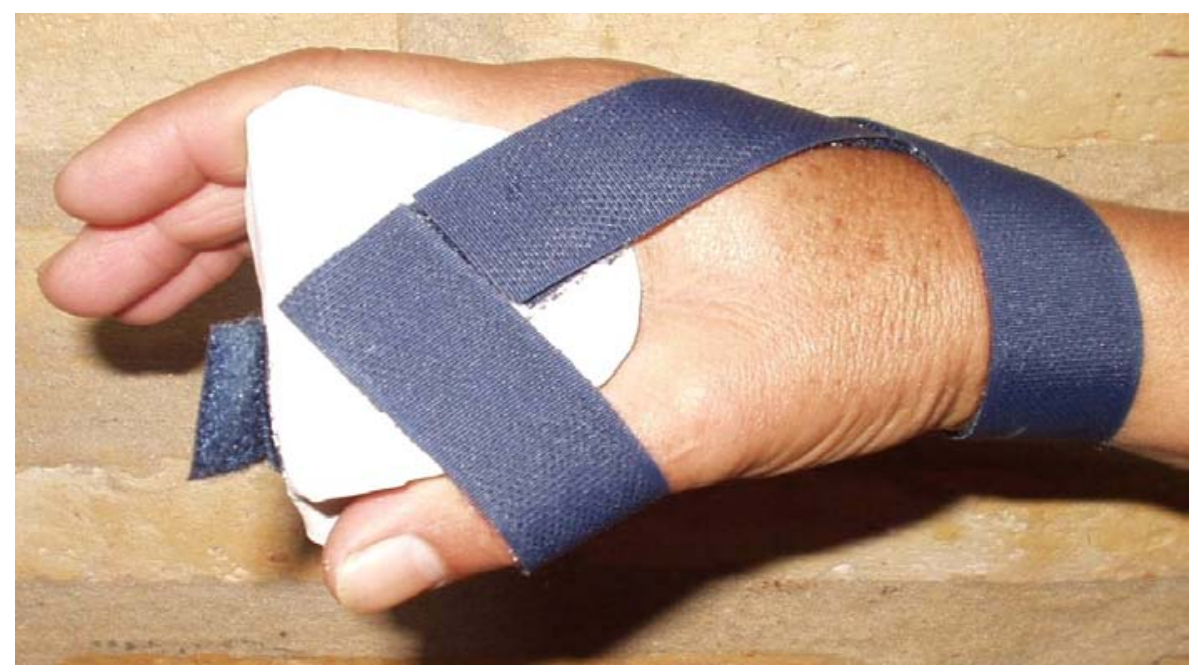


Fotografía 7 - Un abductor del dedo pulgar para un dedo pulgar no funcional

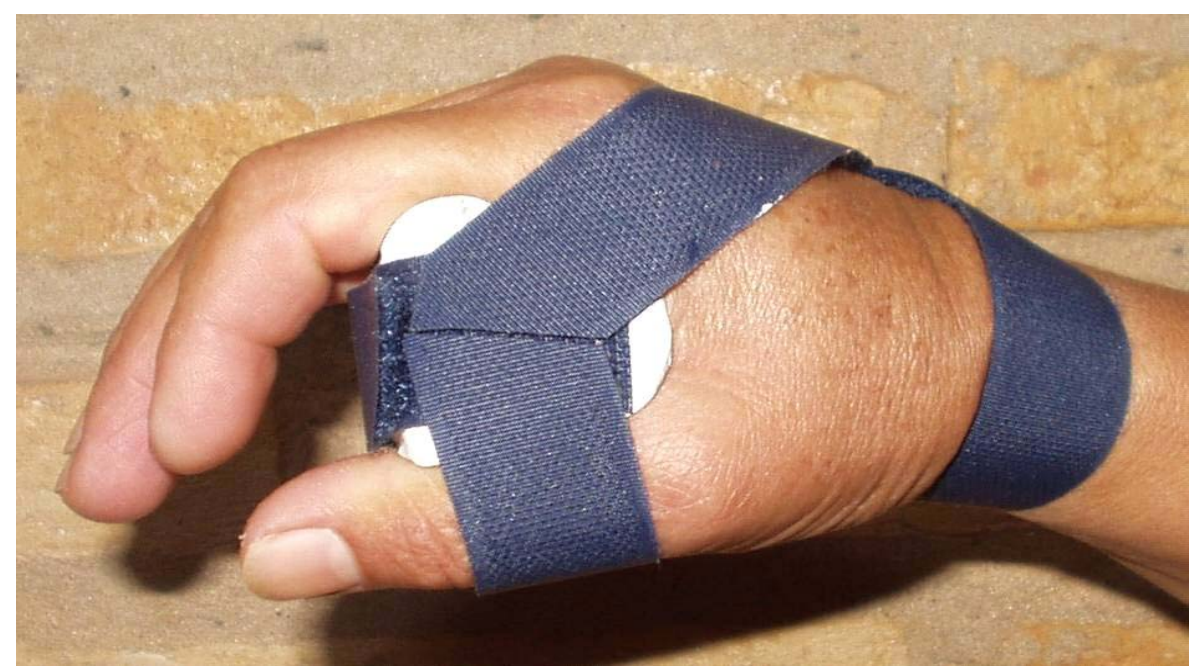

\section{Discusión :}

Esta técnica no es perfecta, y necesita mejoras; yo espero poder realizarlas junto con otros terapeutas ocupacionales. A pesar de ello se describen a continuación algunas de sus ventajas:

COSTO: Su costo no es equivalente al costo de las férulas más baratas (bambú y cuero) pero tienen un precio similar a la férula de venda enyesada y son mucho más baratas que los plásticos de altas temperaturas.

DISPONIBILIDAD LOCAL DE MATERIALES, HERRAMIENTAS Y HABILIDADES: Las herramientas necesarias para elaborar las férulas están disponibles en la mayoría de las comunidades; muchos de los materiales también están disponibles. Gran parte del proceso (cortar la madera, pintar, taladrar los agujeros en la madera, aplicar los tornillos, aplicar el papel) puede ser realizado por un artesano de la comunidad en lugar de ser realizado por un terapeuta; en los países como Paraguay donde los terapeutas tienen el tiempo muy limitado para dedicarse a cada paciente, y los técnicos con estas habilidades son abundantes y baratos, ésta es una consideración importante. La férulas pueden hacerse con herramientas manuales normalmente disponibles en las comunidades paraguayas, aunque el uso de un taladro eléctrico y una sierra acelerarán el proceso.

DUREZA Y DURABILIDAD: El resultado es una férula resistente, que puede ser usada por pacientes afectados por espasticidad y que tiene una durabilidad de aproximadamente seis meses sin requerir mayores reparaciones. Cuando es necesario puede ser reparada fácilmente recortando el papel y retirando la pintura, cortando o encolando sobre el isopor nuevamente y re-aplicando luego el papel y la pintura.

HIGIENE: Las férulas tienen una superficie suave que es muy fácil de limpiar.

FACILIDAD DE MODIFICACIÓN: Algunos problemas (el velcro estropeado, las correas sueltas, las rajaduras en la superficie pintada) pueden ocurrir con el tiempo; un miembro de la familia o un 
artesano de la comunidad pueden reparar estos problemas; ya que los tornillos sostienen las correas, un artesano de la comunidad o un miembro de la familia pueden modificar la longitud de las correas quitando los tornillos y cambiando las correas.

PESO: Estas férulas son más livianas que aquellas de venda de yeso de Paris y se comparan en peso a las de PVC, a las férulas de plástico de baja temperatura y a las de polipropileno.

SE ACOMODA A LA MANO DEL PACIENTE: Las férulas pueden tallarse para encajar bien en la mano del paciente, aún cuando está deformada y/o es espástica.

TIEMPO DE FABRICACIÓN: La parte del proceso de fabricación que el Terapeuta Ocupacional debe realizar (evaluar y medir la mano, ver la forma final del isopor, chequear la férula por completo), requerirá entre 15 y 45 minutos, dependiendo del tipo de férula. Este tiempo es mayor al que se requiere para realizar las férulas de venda de yeso de París o aquellas de polipropileno, y se compara a la elaboración de las férulas de plástico de baja temperatura y las de PVC.

Además, algunos pacientes pueden realizar algunas de las tareas (pintar, empapelar, cortar) para poder, de esta forma involucrarse en la confección de su férula como una actividad terapéutica la cual puede mejorar la coordinación motora, las habilidades de percepción o la destreza de la mano no afectada.

Estas férulas presentan algunas desventajas, las cuales pueden alivianarse a medida que la técnica se va desarrollando y adaptando a las condiciones locales:

- En áreas con pocas industrias, la madera terciada y las planchas de isopor puede que no sean localmente fabricadas por lo tanto pueden resultar demasiado caras para los pacientes.

- En Paraguay, prácticamente no hay ninguna colaboración entre los terapeutas y los artesanos de la comunidad; la comunicación y los sistemas de pago tendrán que ser desarrollados; esto puede tomarles tiempo a los terapeutas, a menos que los hospitales se encarguen de esto.

- Los terapeutas necesitarán desarrollar una nueva forma de pensar; con otros materiales, el terapeuta da forma al material encima de la mano o en un molde de la mano; con esta tecnología, el terapeuta utilizar las técnicas para perfilar el espacio negativo que rodea la mano y formar una férula de acuerdo a ella.

- Durante las estaciones lluviosas, el papel y pintura secarán muy despacio y la producción estará retrasada considerablemente.

- En su forma presente, los pacientes que necesiten tener sus férulas completamente desinfectadas no pueden usar este tipo de férula, ya que no pueden autoclavarse ni pueden se sumergidas en desinfectante.

- La medida de los ángulos de las diferentes articulaciones de la mano es un proceso complejo, normalmente no se realiza por los terapeutas ocupacionales en Paraguay; esto requerirá práctica o cambio del método.

- A pesar de que estas férulas son livianas, la masa de isopor las hace voluminosas y pueden darle al paciente la impresión de ser pesadas. 
Un resumen de ventajas y desventajas de los diferentes materiales para férulas se presenta en la tabla 1.

Tabla 1: RESUMEN DE VENTAJAS Y DESVENTAJAS DE LOS DIFERENTES MATERIALES PARA FÉRULAS

\begin{tabular}{|c|c|c|c|c|c|c|c|}
\hline $\begin{array}{l}\text { Características más } \\
\text { destacadas del Material }\end{array}$ & $\begin{array}{l}\text { Venda } \\
\text { enyesada }\end{array}$ & PVC & Bambú & Cuero & Metal & Poliprop & $\begin{aligned} & \text { Isopor } \\
+ & \text { Madera }\end{aligned}$ \\
\hline Costo & + & 0 & + & + & + & - & + \\
\hline Tiempo de elaboración & + & 0 & 0 & - & - & - & 0 \\
\hline Resistencia & + & + & 0 & - & - & - & + \\
\hline Dureza & - & + & + & + & + & + & + \\
\hline Facilidad para limpiar & - & + & 0 & - & + & + & + \\
\hline Facilidad para modificar & 0 & - & + & + & 0 & - & + \\
\hline $\begin{array}{l}\text { Habilidades y herramientas } \\
\text { localmente disponibles }\end{array}$ & 0 & - & + & + & + & - & + \\
\hline Peso & 0 & + & + & + & - & + & + \\
\hline $\begin{array}{l}\text { Buena adaptación a la mano del } \\
\text { paciente }\end{array}$ & + & - & - & + & 0 & + & + \\
\hline \multicolumn{8}{|c|}{$\begin{array}{l}\text { Referencias (+) ventaja con respecto a otros materiales } \\
\text { (0) neutral } \\
(-) \text { desventaja con respecto a otros materiales }\end{array}$} \\
\hline
\end{tabular}

Hay mucho espacio para la expansión de esta técnica. Hasta ahora, sólo se han hecho férulas estáticas, pero utilizando materiales como las rayas de bicicletas para los tensores (sujetado a la madera terciada), se podría hacer una férula dinámica de extensión de los dedos. Para elaborar otras férulas dónde se necesitan estructuras pequeñas (las férulas activas para evitar desviación cubital, por ejemplo) otros materiales tendrán que ser incorporados y deberán desarrollarse técnicas para poder sujetarlos a la férula, ya que el isopor no tiene la resistencia necesaria para utilizarse en estructuras pequeñas. Éstas son algunas posibilidades. Yo estaría interesado en conocer los trabajos de otras personas al respecto.

He elaborado dos férulas de tobillo-pie utilizando esta técnica haciendo una caja de madera terciada y moldeando el isopor alrededor del pie y en la parte baja de la pierna. Las férulas resultantes fueron satisfactorias para el posicionamiento den cama pero eran demasiado débiles y voluminosas para deambular. También resultó difícil entrar y sacar el pie fuera de la férula y aplicar el papel y la pintura. Un enfoque diferente podría ser realizar una caja de madera terciada que pueda desarmarse y ensamblarse alrededor del pie.

El uso del isopor de embalaje disminuiría el costo y aumentaría el uso potencial de la tecnología. Tendrán que ser desarrollados métodos alternativos para unir los diferentes pedazos, ya que el isopor de embalaje casi nunca tiene los pedazos lo suficientemente grandes como para cortar la férula entera.

Los terapeutas ocupacionales continuarán usando los materiales y habilidades disponibles en sus comunidades para elaborar las férulas de mano. En lugares donde el isopor, la madera terciada y 
el papel están disponibles y otros materiales son demasiado caros, esta tecnología debería ofrecerles a los terapeutas ocupacionales una alternativa atractiva. Una descripción detallada del proceso de fabricación puede obtenerse del autor.

\section{- Referencias}

1) TROMBLY, CA, SCOTT, DC Occupational Therapy for Physical Dysfunction, Williams y Wilkins, Baltimore/London, 1977; 287-288

2) WERNER, D Disabled Village Children, Hesperian Foundation, Berkeley, EE.UU., $199 ; 221$

3) IAFFEI, FATIMA (Terapeuta Ocupacional), Comunicación Personal, Asunción, Paraguay, 2004,

4) HAREAU, J (Terapeuta Ocupacional y Fisioterapista) Comunicación Personal, Asunción, Paraguay, 2003,

5) HOBBS, L, MCDONOUGH, S, O'CALLAGHAN, UN, Life After Injury, Third World Network, Malaysia, 2002, 428,

6) HOBBS, L, MCDONOUGH, S, O'CALLAGHAN, UN, Life After Injury, Third World Network, Malaysia, 2002; 431,432

7) HOBBS, L, MCDONOUGH, S, O'CALLAGHAN, UN, Life After Injury, Third World Network, Malaysia, 2002; 430,431

8) HOBBS, L, MCDONOUGH, S, O'CALLAGHAN, UN, Life After Injury, Third World Network, Malaysia, 2002; 435

9) HOBBS, L, MCDONOUGH, S, O'CALLAGHAN, UN, Life After Injury, Third World Network, Malaysia, 2002; 430,428

10) GONZALEZ, FERNANDO (Técnico Ortopédico), Comunicación Personal, Asunción, Paraguay, 2004,

11) PEDRETTI, LW, Basic Practice Skills in Occupational Therapy for Physical Dysfunction, San José State University, San José, California, EE.UU., 1997; 277 